\title{
Developing a new energy-saving, photosynthesis-promoting environmental control system for greenhouse production based on a heat pump with a heat storage system
}

\author{
Yasunaga IWASAKI*, ${ }^{\dagger}$, Masaki AIZAWA**, Chie YosHIDA**, and Masuyuki TAKAICHI*** \\ $\left(\begin{array}{c}* \text { National Institute of Vegetable and Tea Science, National Agriculture and Food Research Organization, } \\ \text { Minaminakane 40-1, Taketoyo, Aichi, 470-2351, Japan } \\ * * \text { Miyagi Prefectural Institute of Agriculture and Horticulture, } \\ \text { Takadatekawakami Higashikongoji 1, Natori, Miyagi, 981-1243, Japan } \\ * * * \text { National Institute of Vegetable and Tea Science, National Agriculture and Food Research Organization, } \\ \text { Kannondai 3-1-1, Tsukuba, Ibaraki, 305-8666, Japan }\end{array}\right)$
}

\begin{abstract}
We installed an experimental environmental control system, consisting of a heat pump, a fan coil unit, a soil-water heat exchanger, and a heat storage tank, in a greenhouse and investigated its thermal characteristics. The system reduced ventilation time, and the $\mathrm{CO}_{2}$ concentration and humidity in the greenhouse thus remained high. Therefore, promotion of photosynthesis is expected. Its energy consumption was only $45 \%$ of that of a conventional oil-fired air heater.
\end{abstract}

Key words: $\quad \mathrm{CO}_{2}$ enrichment, Coefficient of performance, Energy saving, Environmental control system, Greenhouse, Thermal storage.

\section{Introduction}

To reduce emissions of greenhouse gases and to cope with rapid fluctuations in fuel prices, Japanese greenhouse growers need to reduce their energy consumption. In addition, their income has dropped on account of downturns in the market price of their produce. Thus, new technologies to reduce energy consumption while increasing yield per unit area are needed.

In coastal areas on the Pacific Ocean side of Japan, heated greenhouses must be ventilated during the day, which causes heat loss, even in winter. If the heat could be stored and used for heating at night, energy use and cost could be greatly reduced. Heat storage techniques were investigated extensively when fuel prices soared in the 1980s, and some were introduced into commercial greenhouses (Oguni, 1982; Shoji et al., 1982; Hayashi et al., 1983; Kozai et al., 1983).

Received; July 26, 2012.

Accepted; January 25, 2013.

†Corresponding Author: iwasakiy@affrc.go.jp
However, they fell out of use when fuel prices fell again.

$\mathrm{CO}_{2}$ enrichment in greenhouses promotes photosynthesis by crops (Kawashima, 1991; Kawashiro et al., 2009), yet only $2.7 \%$ of the total greenhouse area in Japan uses it (MAFF, 2009). Ventilation defeats the purpose of $\mathrm{CO}_{2}$ enrichment, so the effect on yield is often unclear. Any technique that avoids the need for ventilation would improve the effect of $\mathrm{CO}_{2}$ enrichment.

We have developed a new environmental control system that uses a heat pump to store heat, thus reducing energy consumption, avoiding the need for ventilation and so promoting photosynthesis. To improve efficiency of energy use in the heat storage/release process, we designed the system using a fan coil unit and a soil heat exchanger in combination with a heat pump. Here, we evaluated the system's thermal and energy-saving properties, and investigated the effects on the environment in a greenhouse, especially on $\mathrm{CO}_{2}$ concentration and relative humidity, which greatly affect photosynthesis. 


\section{Materials and Methods}

\subsection{Greenhouses and experimental setup}

In two north-south-oriented greenhouses, a conventional oil-fired air heater installed at the northern end supplied hot air via two plastic ducts laid on the ground along both side walls (Fig. 1). In one greenhouse, designated the experimental greenhouse $\left(\mathrm{G}_{\mathrm{ex}}\right)$, we installed our environmental control system. The other was designated the control greenhouse $\left(\mathrm{G}_{\mathrm{ct}}\right)$.

Both were single-span, saddle-roof greenhouses of $12 \mathrm{~m}$ long, $6.3 \mathrm{~m}$ wide, and $3.3 \mathrm{~m}$ high, made of steel pipes covered with polyolefin film. The internal thermal screens were made of polyethylene film. The thermal screens of the roofs and side walls were opened during the daytime (0800h-1700h) and closed during the night (1700h-0800h). The side windows of the greenhouses were opened and closed for ventilation depending on the room air temperature.

The experiment ran from March 10 to April 9, 2009 in Natori City, Japan $\left(38.17^{\circ} \mathrm{N}\right)$.

\subsection{Environmental control system}

The environmental control system consists of a heat storage tank, a heat pump, a fan coil unit, a soil-water heat-exchange unit, and three circulation pumps (Fig. 2). We also installed an auxiliary oil air heater, a $\mathrm{CO}_{2}$ generator, and various sensors.

The heat storage tank was made of $10-\mathrm{mm}$ plywood insulated with $100-\mathrm{mm}$ polystyrene foam, and waterproofed with 2-mm-thick plastic sheeting. It formed a rectangular parallelepiped of $2.7 \mathrm{~m}$ long, $0.9 \mathrm{~m}$ wide, and $1.2 \mathrm{~m}$ deep, with a capacity of $2916 \mathrm{~L}$. It was installed underground inside the northern end of the greenhouse and filled with water.

An air-water heat pump (YWYP125A, Daikin, Osaka, Japan; $3.75 \mathrm{~kW}$ rated electric power consumption, $13.5 \mathrm{~kW}$ heating capacity, $11.2 \mathrm{~kW}$ cooling capacity) and a fan coil unit (UAHM90B water-air heat exchanger, Daikin; $0.4 \mathrm{~kW}$ rated electric power consumption) were equipped with three water circulation pumps (WUP3-325-0.15S, Kawamoto Pump, Aichi, Japan; $0.15 \mathrm{~kW}$ rated power output). Each circulation pump ran simultaneously with the heat pump or the fan

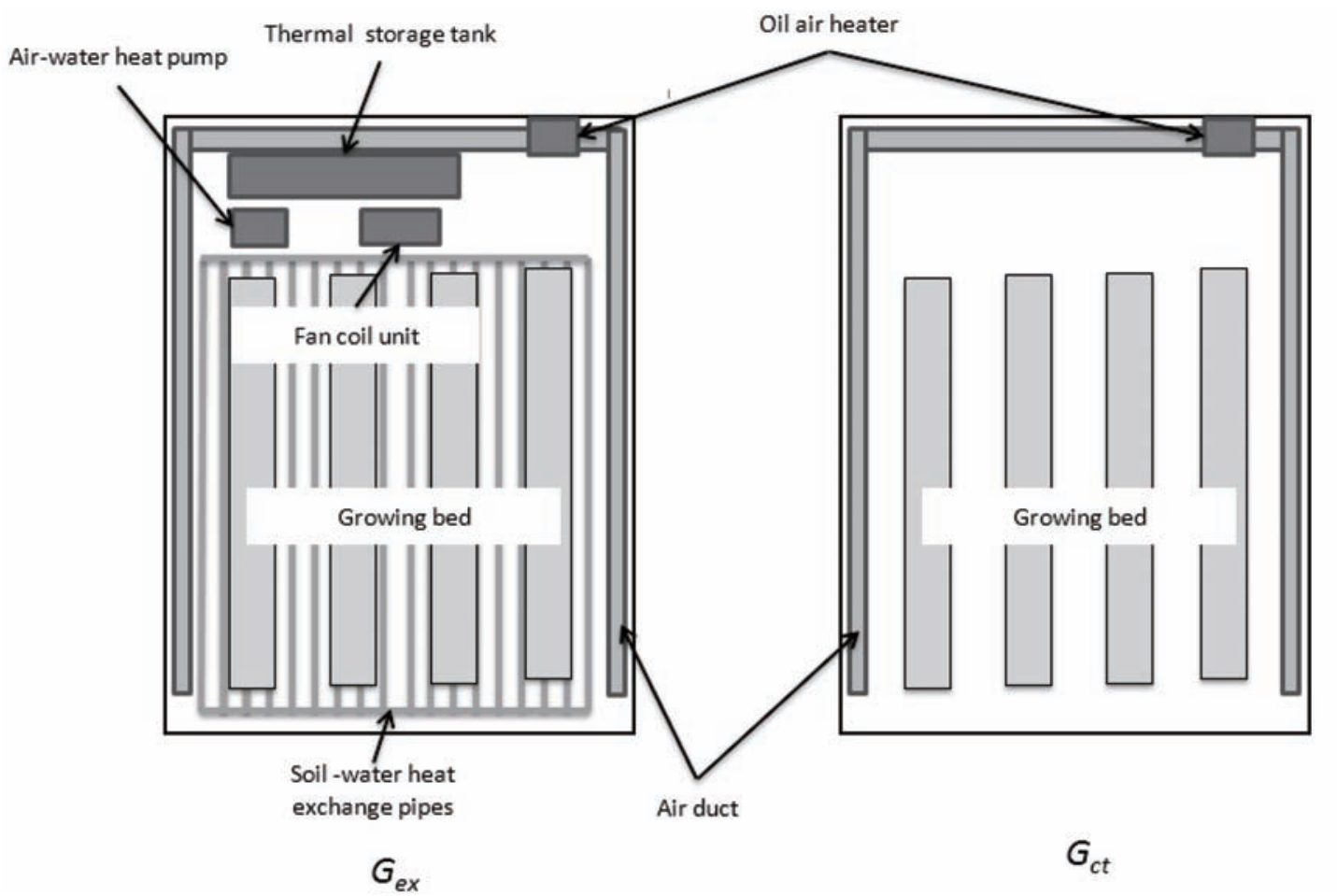

Fig. 1. Schematic diagram of the experimental greenhouses. Gex: greenhouse with experimental environmental control system; Gct: greenhouse with conventional oil heater. 


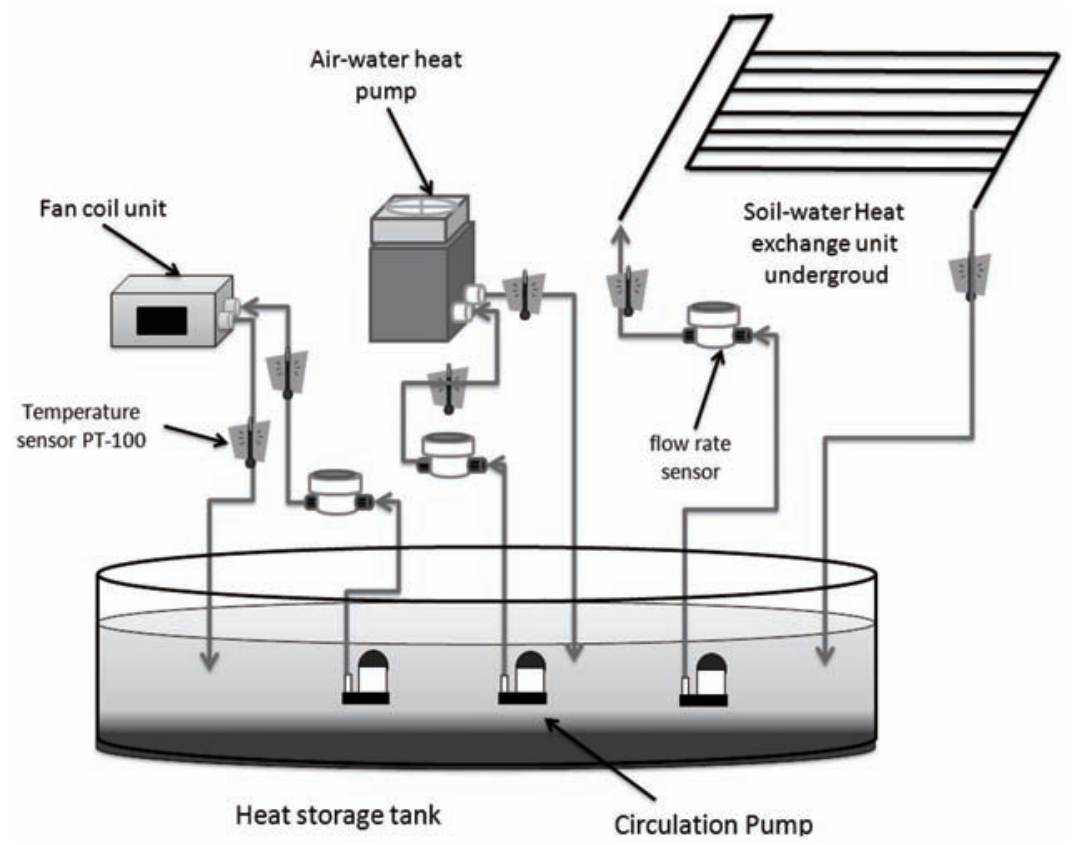

Fig. 2. Schematic diagram of the experimental environmental control system.

coil unit. Polyethylene pipes (27 mm wide, $1 \mathrm{~mm}$ thick) were buried $0.3 \mathrm{~m}$ deep, $0.3 \mathrm{~m}$ apart, in the soil in the greenhouse as a soil-water heat exchanger (with a surface area of $8.0 \mathrm{~m}^{2}$ and a water capacity of $56 \mathrm{~L}$ ). A programmable logic controller (KV-3000 series, Keyence, Osaka, Japan) controlled the system.

An oil-fired air heater (KA-205, Nepon Inc., Tokyo, Japan; $23.3 \mathrm{~kW}$ ) was installed in each greenhouse, and kerosene consumption was measured with an oil meter (RE10LF, Nitto Seiko, Kyoto, Japan). A CO 2 generator $\left(0.3 \mathrm{~L} \mathrm{~h}^{-1}\right.$ kerosene) ran continuously from $0830 \mathrm{~h}$ to $1200 \mathrm{~h}$ every day in each greenhouse.

When the daytime air temperature exceeded $25^{\circ} \mathrm{C}$, the environmental control system removed heat from inside the greenhouse and stored it in the heat storage tank and the soil of the greenhouse floor. When the air temperature fell below $23^{\circ} \mathrm{C}$, it stopped. When the air temperature exceeded $30^{\circ} \mathrm{C}$, the system opened the side windows for ventilation. When the nighttime air temperature fell below $15^{\circ} \mathrm{C}$, the stored heat was piped in, and when it exceeded $17^{\circ} \mathrm{C}$, the system stopped.

During cooling, the system selected the fan coil unit when the water temperature in the heat storage tank was $<15^{\circ} \mathrm{C}$ and selected the heat pump when the water temperature was $\geq 15^{\circ} \mathrm{C}$. When the water temperature reached $45^{\circ} \mathrm{C}$, it stopped. When the temperature difference between the water in the tank and the soil at a depth of $20 \mathrm{~cm}$ (between the heat-exchange pipes) was $>10^{\circ} \mathrm{C}$, the system started circulating the hot water into the soil-water heat exchanger, and when the difference was $\leq 5^{\circ} \mathrm{C}$, it stopped. During heating, when the water temperature in the tank was $\geq 20^{\circ} \mathrm{C}$, the system used the fan coil to release heat, and when the water temperature was $<20^{\circ} \mathrm{C}$, it used the heat pump. Some of the heat stored in the soil radiated directly from the soil surface into the greenhouse at night. When the outlet water temperature of the heat pump decreased to $\leq 5^{\circ} \mathrm{C}$, the system stopped the heat pump to prevent damage by freezing, and the auxiliary air heater supplied heat.

\subsection{Measurements}

The air temperature inside and outside the greenhouses was measured by platinum resistance thermometers (RD-10M, Shimaden Co., Ltd. , Tokyo, Japan; Pt100) with an aspirated tube radiation shield. The inside sensors were set at 0.5 and $1.0 \mathrm{~m}$ above the ground in the center of the greenhouse. The inside relative humidity $(\mathrm{RH})$ was measured by a humidity sensor (ES2-HB, Omron, Kyoto, Japan). The $\mathrm{CO}_{2}$ concentration inside was measured by a $\mathrm{CO}_{2}$ sensor (MA-10, Chino, Yamagata, Japan). The mass flow rate of the water in the pipes was measured by flow 
meters (VWK30RC pulse transmitter, Kimmon Co., Tokyo, Japan). The temperature of the water entering and leaving the heat pump, fan coil unit, and soil-water heat exchanger was measured by platinum resistance thermometers (RD-10M) mounted on the inlet and outlet lines. The electrical power input into the heat pump, fan coil unit, and circulating pumps was measured by wattmeters (Clamp On Power HiTESTER 3168, Hioki E. E. Corp., Nagano, Japan). The ground temperatures at depths of 0.1 and $0.2 \mathrm{~m}$ were measured by platinum resistance thermometers (RD-10M). The solar radiation inside and outside the greenhouse was measured by a pyranometer (MS-601, EKO Instruments Co. Ltd., Tokyo, Japan). All data from the sensors were automatically recorded in the programmable logic controller every $60 \mathrm{~s}$.

\subsection{Plants}

Paprika (Capsicum annuum cv. Fiesta) plants were grown hydroponically during the experiment. Seedlings at the flowering stage were transplanted at a planting density of 2.7 plants $\mathrm{m}^{-2}$ in both greenhouses on January 17, 2008. The soil surfaces of the greenhouses were covered with black non-woven cloth.

\section{Results}

\subsection{Environmental measurements}

Environmental measurement data are summarized from $0600 \mathrm{~h}$ of one day to $0559 \mathrm{~h}$ of the next day (Table 1). Average minimum and maximum air temperatures were 14.9 and $31.5^{\circ} \mathrm{C}$ inside and 1.4 and $14.3^{\circ} \mathrm{C}$ outside, respectively. Average minimum and maximum water temperatures in the heat storage tank were 12.5 and $41.1^{\circ} \mathrm{C}$, respectively. Average minimum and maximum soil temperatures were 20.4 and $26.5^{\circ} \mathrm{C}$ at $0.1 \mathrm{~m}$ and 21.7 and $26.8^{\circ} \mathrm{C}$ at $0.2 \mathrm{~m}$, respectively. Average minimum and maximum inside relative humidities were 34.8 and $75.5 \%$, respectively.

\subsection{Thermal performance}

The daily solar radiation during the experiment averaged 2,382 $\mathrm{Wh} \mathrm{m}^{-2}$. Only on one day (March 24) was the solar radiation insufficiently high to warrant cooling. The average heat energy removed was 879 Wh m $\mathrm{m}^{-2}: 823 \mathrm{Wh} \mathrm{m}^{-2}(93.6 \%)$ by the heat pump and $57 \mathrm{Wh} \mathrm{m}^{-2}(6.5 \%)$ by the fan coil unit. The heat pump, which was more effective, dominated the cooling because the primary purpose of cooling was to reduce the ventilation time. Of the heat energy removed, $445 \mathrm{Wh}$ $\mathrm{m}^{-2}$ was transferred into the soil by the soil-water heat- exchange unit. The electricity consumption during cooling averaged $309 \mathrm{Wh} \mathrm{m}^{-2}$ per day (Table 2).

At night, the average heat energy released was 444 Wh $\mathrm{m}^{-2}: 251 \mathrm{Wh} \mathrm{m}^{-2}(56.5 \%)$ by the heat pump and $193 \mathrm{Wh} \mathrm{m}^{-2}(43.5 \%)$ by the fan coil unit. The electricity consumption during heating averaged $97 \mathrm{Wh}$ $\mathrm{m}^{-2}$ (Table 3).

The outlet water temperature of the heat pump at midnight was $<5^{\circ} \mathrm{C}$ (and the heat pump stopped) on 15 days during the experiment. At these times, the air heater cut in. The average heat energy supplied by the heater was $111 \mathrm{Wh} \mathrm{m}^{-2}$ per day (Table 3).

\subsection{Supply of heat from the soil surface to the air inside the greenhouse}

The average heat load, $Q_{\text {load.h }}$, was $1,139 \mathrm{Wh} \mathrm{m}^{-2}$ per day, calculated from the consumption of kerosene by the air heater in $\mathrm{G}_{\mathrm{ct}}$ and the average heating degree hour of $182^{\circ} \mathrm{C} \cdot \mathrm{h}$ day $^{-1}$ during the experiment (Table 3 ). In $G_{\text {ex }}$, the total amount of heat released from the fan coil unit, the heat pump, and the air heater, $Q_{\text {total.h }}$, averaged $556 \mathrm{Wh} \mathrm{m}^{-2}$. We presume that the difference between $Q_{\text {total.h }}$ and $Q_{\text {load.h }}$ is the amount of heat radiated from the soil surface to the air inside the greenhouse $\left(Q_{\text {soil.h }}\right)$, which was $583 \mathrm{Wh} \mathrm{m}^{-2}$ per day. The amount of heat transferred from the heat storage tank to the soil during the daytime, $Q_{\text {soil.c, }}$ averaged $445 \mathrm{Wh} \mathrm{m}^{-2}$ per day. The difference between $Q_{\text {soil.h }}$ and $Q_{\text {soil.c, }}, 138 \mathrm{Wh}$ $\mathrm{m}^{-2}$ per day, was therefore the heat transferred directly from the air to the soil surface or from solar radiation to the soil surface.

\subsection{Coefficient of performance of the system}

The coefficient of performance (COP) during cooling, $\varepsilon_{c}$, ranged from 2.1 to 14.5 (mean, 3.3; Table 4). COP during heating, $\varepsilon_{\mathrm{h}}$, ranged from 2.7 to 6.4 (mean, 4.6). If the purpose of the cooling is to store the heat for release at nighttime, calculation of the COP for heating should include the electricity consumed during cooling. In this case, overall COP, $\varepsilon_{\mathrm{t} 1}$, ranged from 0.3 to 5.7 (mean, 1.4). COP $\varepsilon_{t 1}$ increased when there was less daytime cooling and it decreased when there was less nighttime heating, since cooling consumed more energy than heating.

For the COP of the heat pump, during cooling, the water temperature at the inlet of the heat pump influenced the COP: a higher inlet water temperature reduced the COP (Fig. 3). During heating, the COP decreased with decreasing temperature of the inlet water to the heat pump (operated at $<20^{\circ} \mathrm{C}$ ) (Fig. 4) . 
Table 1. Daily maximum and minimum air temperatures inside and outside the greenhouse, and of the heat storage tank.

\begin{tabular}{|c|c|c|c|c|c|c|c|c|c|c|c|c|}
\hline \multirow[t]{2}{*}{$\begin{array}{c}\text { Date } \\
\text { 0600h-0559h } \\
\text { next day }\end{array}$} & \multicolumn{2}{|c|}{$\begin{array}{l}\text { Inside } \\
\text { air temp. } \\
\left({ }^{\circ} \mathrm{C}\right)\end{array}$} & \multicolumn{2}{|c|}{$\begin{array}{l}\text { Outside } \\
\text { air temp. } \\
\quad\left({ }^{\circ} \mathrm{C}\right)\end{array}$} & \multicolumn{2}{|c|}{$\begin{array}{l}\text { Water te mp. } \\
\text { in heat } \\
\text { storage tank } \\
\left({ }^{\circ} \mathrm{C}\right)\end{array}$} & \multicolumn{2}{|c|}{$\begin{array}{l}\text { Soil temp. at } \\
0.1 \text { m depth } \\
\left.{ }^{\circ} \mathrm{C}\right)\end{array}$} & \multicolumn{2}{|c|}{$\begin{array}{l}\text { Soil temp. } \\
\text { at } 0.2 \mathrm{~m} \\
\text { depth } \\
\left({ }^{\circ} \mathrm{C}\right)\end{array}$} & \multicolumn{2}{|c|}{$\begin{array}{c}\text { Inside relative } \\
\text { humidity } \\
(\%)\end{array}$} \\
\hline & Min & Max & Min & Max & Min & Max & Min & Ma & Min & Max & Min & Max \\
\hline \multicolumn{13}{|l|}{ March } \\
\hline $10-11$ & 14.5 & 31.8 & 0.5 & 16.0 & 11.5 & 44.6 & 18.6 & 24.9 & 19.4 & 25.3 & 31.6 & 74.0 \\
\hline $11-12$ & 13.8 & 29.9 & -0.7 & 6.8 & 11.7 & 40.4 & 18.7 & 24.0 & 19.9 & 24.2 & 27.9 & 68.1 \\
\hline $12-13$ & 4.5 & 32.7 & -1.7 & 10.3 & 10.8 & 44.4 & 18.4 & 25.2 & 19.6 & 25.6 & 29.8 & 72.3 \\
\hline $13-14$ & 14.1 & 29.0 & -0.2 & 13.7 & 10.8 & 37.5 & 19.4 & 23.6 & 20.5 & 24.0 & 35.2 & 82.7 \\
\hline $14-15$ & 14.4 & 28.8 & 2.7 & 12.5 & 11.2 & 33.6 & 18.4 & 21.5 & 20.0 & 22.3 & 34.0 & 84.0 \\
\hline $15-16$ & 4.3 & 35.8 & 0.6 & 13.9 & 11.6 & 44.4 & 18.0 & 25.8 & 19.2 & 25.9 & 26.7 & 81.5 \\
\hline $16-17$ & 5.9 & 30.8 & 0.8 & 17.8 & 15.6 & 44.6 & 20.5 & 25.1 & 21.4 & 25.9 & 38.9 & 83.3 \\
\hline $17-18$ & 5.2 & 27.5 & 0.5 & 14.8 & .2 & 4.6 & 20.3 & 24.4 & .3 & 25.7 & 42.7 & 78.0 \\
\hline $18-19$ & 5.9 & 40.4 & 1.1 & 17.0 & 11.7 & 44.3 & 20.0 & 27.8 & 21.2 & 27.6 & 38.4 & 83.0 \\
\hline $19-20$ & 6.1 & 40.2 & 8.2 & 22.5 & 25.9 & 44.3 & 22.4 & 30.1 & 23.6 & 29.5 & 32.8 & 86.4 \\
\hline $20-21$ & 5.2 & 32.7 & 2.2 & 13.8 & 11.8 & 44.5 & 21.9 & 27.2 & 9 & 28.1 & 30.3 & 85.2 \\
\hline $21-22$ & 4.3 & 23.8 & 3.2 & 12.8 & 9.9 & 13.0 & 19.1 & 21.4 & 0.3 & 21.6 & 57.7 & 84.8 \\
\hline $22-23$ & 15.0 & 29.2 & -0.9 & 12.6 & 10.0 & 44.7 & 18.8 & 25.6 & 19.9 & 26.2 & 36.7 & 69.8 \\
\hline $23-24$ & 5.9 & 32.0 & 1.2 & 10.5 & 11.1 & 44.4 & 20.2 & 27.4 & 21 & 27.2 & 34.8 & 77.6 \\
\hline $24-25$ & 3.9 & 17.8 & 0.4 & 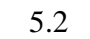 & 9.8 & 17.8 & 17.9 & 21.8 & .8 & 23.4 & 54.0 & 69.3 \\
\hline $25-26$ & 13.8 & 29.4 & -2.9 & 10.0 & 9.9 & 44.8 & 17.7 & 24.6 & 19.3 & 25.5 & 40.7 & 69.0 \\
\hline $26-27$ & 14.1 & 32.7 & -1.5 & 10.9 & 11.7 & 44.3 & 19.1 & 26.5 & 20.7 & 26.5 & 36.6 & 64.2 \\
\hline $27-28$ & 4.6 & 30.6 & -1.4 & 11.0 & 11.5 & 44.2 & 19.9 & 26.1 & 21.3 & 26.5 & 35.5 & 70.9 \\
\hline $28-29$ & 15.9 & 31.6 & -0.5 & 10.6 & 11.3 & 44.5 & 20.3 & 27.1 & 21.7 & 27.4 & 38.0 & 72.0 \\
\hline 29-30 & 14.8 & 31.0 & -1.4 & 14.3 & 13.3 & 44.6 & 21.3 & 26.8 & 22.4 & 27.4 & 36.0 & 74.1 \\
\hline $30-31$ & 6.1 & 31.1 & 0.6 & 14.1 & 11.6 & 44.3 & 21.0 & 27.6 & 22.3 & 27.9 & 38.7 & 76.7 \\
\hline \multicolumn{13}{|l|}{ April } \\
\hline $31-1$ & 4.4 & 21.1 & 3.3 & 5.7 & 9.9 & 23.3 & 18.6 & 22.2 & 20.4 & 23.9 & 58.7 & 72.6 \\
\hline $1-2$ & 5.2 & 31.4 & 1.7 & 13.6 & 10.0 & 44.5 & 18.5 & 26.8 & 20.1 & 26.8 & 32.8 & 70.7 \\
\hline $2-3$ & 15.9 & 33.3 & 2.2 & 19.4 & 12.8 & 44.6 & 20.9 & 28.6 & 22.1 & 28.4 & 21.6 & 80.1 \\
\hline $3-4$ & 15.6 & 31.3 & 6.5 & 15.3 & 21.7 & 44.5 & 22.2 & 27.3 & 23.6 & 28.2 & 40.6 & 83.0 \\
\hline $4-5$ & 13.8 & 34.9 & 2.0 & 20.4 & 12.7 & 44.4 & 22.3 & 29.9 & 23.9 & 29.6 & 18.9 & 71.6 \\
\hline 5-6 & 14.1 & 35.2 & 3.1 & 17.7 & 12.5 & 44.5 & 22.9 & 31.2 & 24.3 & 30.3 & 22.9 & 66.7 \\
\hline 6-7 & 14.5 & 35.1 & 4.2 & 22.5 & 12.7 & 44.4 & 24.0 & 31.1 & 25.0 & 30.5 & 23.2 & 74.1 \\
\hline $7-8$ & 16.6 & 34.8 & 3.9 & 18.9 & 13.0 & 44.5 & 24.7 & 31.6 & 25.5 & 30.9 & 22.6 & 66.7 \\
\hline $8-9$ & 15.8 & 39.1 & 5.0 & 25.3 & 12.9 & 44.3 & 24.6 & 32.2 & 25.6 & 31.3 & 26.6 & 72.7 \\
\hline verage & 14.9 & 31.5 & 1.4 & 14.3 & 12.5 & 41.1 & 20.4 & 26.5 & 21.7 & 26.8 & 34.8 & 75.5 \\
\hline
\end{tabular}


Table 2. Heat and electric energy flux measurements during daytime.

\begin{tabular}{|c|c|c|c|c|c|c|}
\hline \multirow{2}{*}{$\begin{array}{c}\text { Date } \\
\text { 0600h-1759h } \\
\end{array}$} & \multirow{2}{*}{$\begin{array}{c}\text { Solar radiation } \\
\text { inside } \\
\mathrm{H} \\
\end{array}$} & \multirow{2}{*}{$\begin{array}{c}\text { Heat removed } \\
\text { by HP } \\
\text { Q } \\
\mathrm{hp,c}\end{array}$} & \multicolumn{2}{|c|}{$\begin{array}{c}\text { Heat removed } \\
\text { by FC } \\
\left(\mathrm{Wh} \mathrm{m}^{-2}\right)\end{array}$} & \multirow{2}{*}{$\begin{array}{c}\text { Heat stored } \\
\text { in soil } \\
\text { Q } \\
\text { Qsoil.c }\end{array}$} & \multirow{2}{*}{$\begin{array}{c}\text { Electricity } \\
\text { consumption } \\
\mathrm{Q}_{\mathrm{e}, \mathrm{c}} \\
\end{array}$} \\
\hline & & & $\mathrm{Q}_{\mathrm{fc}, \mathrm{c}}$ & $\mathrm{Q}_{\mathrm{hp}+\mathrm{fc}, \mathrm{c}}$ & & \\
\hline \multicolumn{7}{|l|}{ March } \\
\hline 10 & 1,985 & 1,063 & 0 & 1,063 & 606 & 329 \\
\hline 11 & 2,065 & 763 & 64 & 827 & 443 & 289 \\
\hline 12 & 2,423 & 1,065 & 57 & 1,122 & 595 & 393 \\
\hline 13 & 1,889 & 589 & 101 & 691 & 380 & 240 \\
\hline 14 & 1,005 & 187 & 0 & 187 & 260 & 46 \\
\hline 15 & 2,706 & 1,101 & 73 & 1,174 & 591 & 349 \\
\hline 16 & 1,608 & 805 & 0 & 805 & 558 & 297 \\
\hline 17 & 1,595 & 833 & 0 & 833 & 518 & 310 \\
\hline 18 & 2,594 & 1,098 & 60 & 1,158 & 514 & 360 \\
\hline 19 & 2,599 & 694 & 0 & 694 & 736 & 284 \\
\hline 20 & 1,489 & 461 & 0 & 461 & 547 & 173 \\
\hline 21 & 797 & 0 & 27 & 27 & 0 & 2 \\
\hline 22 & 2,621 & 1,029 & 125 & 1,154 & 376 & 401 \\
\hline 23 & 2,631 & 967 & 79 & 1,046 & 388 & 343 \\
\hline 24 & 432 & 0 & 0 & 0 & 0 & 0 \\
\hline 25 & 2,910 & 1,049 & 125 & 1,174 & 405 & 389 \\
\hline 26 & 2,937 & 988 & 25 & 1,011 & 407 & 344 \\
\hline 27 & 2,631 & 886 & 112 & 998 & 418 & 347 \\
\hline 28 & 2,027 & 1,008 & 146 & 1,153 & 466 & 385 \\
\hline 29 & N.M & 1,008 & 11 & 1,019 & 433 & 332 \\
\hline 30 & N.M & 984 & 137 & 1,121 & 453 & 364 \\
\hline \multicolumn{7}{|l|}{ April } \\
\hline 1 & N.M & N.M & N.M & N.M & N.M & N.M \\
\hline 2 & 3,156 & 914 & 203 & 1,117 & 465 & 342 \\
\hline 3 & 3,502 & 1,002 & 58 & 1,060 & 506 & 410 \\
\hline 4 & 1,999 & 705 & 0 & 705 & 555 & 330 \\
\hline 5 & 3,304 & 731 & 0 & 731 & 531 & 342 \\
\hline 6 & 3,519 & 964 & 67 & 1,031 & 427 & 385 \\
\hline 7 & 3,086 & 953 & 86 & 1,039 & 443 & 381 \\
\hline 8 & 3,242 & 985 & 0 & 985 & 455 & 393 \\
\hline 9 & 3,557 & 1,024 & 90 & 1,114 & 417 & 389 \\
\hline Average & 2,382 & 823 & 57 & 879 & 445 & 309 \\
\hline
\end{tabular}

N.M : Not Measured

HP : heat pump FC : fan coil unit 
Y. Iwasaki et al. : Developing a new energy-saving, photosynthesis-promoting environmental control system

Table 3. Heat and electric energy flux measurements during nighttime.

\begin{tabular}{|c|c|c|c|c|c|c|c|c|c|}
\hline \multirow[t]{2}{*}{$\begin{array}{c}\text { Date } \\
\text { 1800h-0559h } \\
\text { next day }\end{array}$} & $\begin{array}{c}\text { Heat } \\
\text { released } \\
\text { from HP }\end{array}$ & $\begin{array}{l}\text { Heat } \\
\text { released } \\
\text { from FC }\end{array}$ & $\begin{array}{l}\text { Heat } \\
\text { released } \\
\text { from } \\
\text { HP\&FC }\end{array}$ & $\begin{array}{l}\text { Electricity } \\
\text { consum } \\
\text { ption } \mathrm{f} \\
\quad \text { (Wh n }\end{array}$ & \begin{tabular}{l}
\multicolumn{1}{c}{ Heat } \\
relesed \\
from AH \\
$\mathrm{m}^{-2}$ )
\end{tabular} & $\begin{array}{c}\begin{array}{c}\text { Heat } \\
\text { released } \\
\text { from }\end{array} \\
\text { HP, FC, AH }\end{array}$ & $\begin{array}{l}\text { Heat } \\
\text { released } \\
\text { from } \\
\text { the soil }\end{array}$ & \multirow{2}{*}{$\begin{array}{l}\text { Heating } \\
\text { load } \\
\text { Q }_{\text {load.h }} \\
\end{array}$} & \multirow{2}{*}{$\begin{array}{c}\text { Heating } \\
\text { degree } \\
\text { hour } \\
\left({ }^{\circ} \mathrm{C} \cdot \text { hr day }^{-1}\right) \\
D H_{n}\end{array}$} \\
\hline & $\mathrm{Q}_{\mathrm{hp}, \mathrm{h}}$ & $\mathrm{Q}_{\mathrm{fc}, \mathrm{h}}$ & $\mathrm{Q}_{\mathrm{hp}+\mathrm{fc}, \mathrm{h}}$ & $\mathrm{Q}_{\mathrm{e}, \mathrm{h}}$ & $\mathrm{Q}_{\mathrm{ah.h}}$ & $\mathrm{Q}_{\text {total.h }}$ & $\mathrm{Q}_{\text {soil.h }}$ & & \\
\hline \multicolumn{10}{|l|}{ March } \\
\hline $10-11$ & 378 & 218 & 596 & 151 & 251 & 847 & 539 & 1,386 & 192 \\
\hline $11-12$ & 333 & 178 & 512 & 147 & 371 & 882 & 621 & 1,503 & 225 \\
\hline $12-13$ & 400 & 223 & 623 & 122 & 78 & 701 & 710 & 1,411 & 235 \\
\hline $13-14$ & 0 & 76 & 76 & 28 & 0 & 76 & 646 & 722 & 82 \\
\hline $14-15$ & 375 & 125 & 500 & 139 & 218 & 718 & 832 & 1,550 & 181 \\
\hline $15-16$ & 186 & 195 & 381 & 111 & 0 & 381 & 824 & 1,205 & 187 \\
\hline $16-17$ & 168 & 172 & 340 & 100 & 0 & 340 & 829 & 1,170 & 149 \\
\hline $17-18$ & 323 & 211 & 535 & 106 & 0 & 535 & 1,128 & 1,662 & 186 \\
\hline $18-19$ & 0 & 124 & 124 & 37 & 0 & 124 & 641 & 765 & 131 \\
\hline $19-20$ & N.M & N.M & N.M & N.M & N.M & N.M & N.M & 306 & 64 \\
\hline $20-21$ & 370 & 230 & 600 & 138 & 299 & 899 & 716 & 1,616 & 174 \\
\hline $21-22$ & 289 & 0 & 289 & 60 & 185 & 474 & 161 & 636 & 97 \\
\hline $22-23$ & 379 & 240 & 619 & 118 & 19 & 638 & 651 & 1,289 & 201 \\
\hline $23-24$ & 196 & 198 & 394 & 94 & 0 & 394 & 807 & 1,201 & 186 \\
\hline $24-25$ & 404 & 0 & 404 & 71 & 1,142 & 1,547 & 200 & 1,746 & 211 \\
\hline $25-26$ & 352 & 236 & 588 & 137 & 284 & 872 & 687 & 1,559 & 238 \\
\hline $26-27$ & 379 & 269 & 648 & 111 & 119 & 768 & 631 & 1,399 & 219 \\
\hline $27-28$ & 367 & 251 & 618 & 113 & 33 & 651 & 653 & 1,304 & 226 \\
\hline $28-29$ & 244 & 258 & 502 & 91 & 8 & 510 & 779 & 1,289 & 222 \\
\hline $29-30$ & 342 & 240 & 582 & 113 & 19 & 602 & 767 & 1,369 & 226 \\
\hline $30-31$ & 3 & 262 & 265 & 42 & 0 & 265 & 856 & 1,122 & 189 \\
\hline \multicolumn{10}{|l|}{ April } \\
\hline $31-1$ & N.M & N.M & N.M & N.M & N.M & N.M & N.M & N.M & N.M \\
\hline $1-2$ & 294 & 242 & 536 & 96 & 5 & 541 & 690 & 1,231 & 189 \\
\hline $2-3$ & 1 & 203 & 204 & 37 & 0 & 204 & 641 & 846 & 168 \\
\hline $3-4$ & 0 & 139 & 139 & 23 & 0 & 139 & 542 & 681 & 131 \\
\hline $4-5$ & 305 & 241 & 546 & 124 & 81 & 627 & 249 & 876 & 186 \\
\hline $5-6$ & 314 & 230 & 544 & 119 & 0 & 544 & 324 & 868 & 202 \\
\hline $6-7$ & 158 & 213 & 371 & 86 & 0 & 371 & 361 & 732 & 197 \\
\hline 7-8 & 302 & 225 & 528 & 124 & 0 & 528 & 254 & 781 & 203 \\
\hline 8-9 & 164 & 218 & 382 & 82 & 0 & 382 & 430 & 811 & 181 \\
\hline Average & 251 & 193 & 444 & 97 & 111 & 556 & 583 & 1,139 & 182 \\
\hline
\end{tabular}

N.M : Not Measured

$\mathrm{HP}$ : heat pump, FC : fan coil unit, AH : hot air heater 
Table 4. Coefficient of performance (COP) of the system, daily solar energy collection rate, and energy use ratio.

\begin{tabular}{|c|c|c|c|c|c|c|}
\hline Date & $\begin{array}{c}C O P \text { for } \\
\text { cooling } \\
\mathrm{Q}_{\mathrm{hp}+\mathrm{fc}, \mathrm{c}} / \mathrm{Q}_{\mathrm{e}, \mathrm{c}}\end{array}$ & $\begin{array}{c}C O P \text { for } \\
\text { heating } \\
\mathrm{Q}_{\mathrm{hp}+\mathrm{fc}, \mathrm{h}} / \mathrm{Q}_{\mathrm{e}, \mathrm{h}}\end{array}$ & $\begin{array}{c}\text { COP. for } \\
\text { total1 } \\
\mathrm{Q}_{\mathrm{hp}+\mathrm{fc}, \mathrm{h}} \\
/\left(\mathrm{Q}_{\mathrm{e}, \mathrm{c}+\mathrm{Qe}, \mathrm{h}}\right)\end{array}$ & 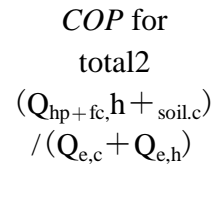 & $\begin{array}{c}\text { Solar energy } \\
\text { collecting rate } \\
\mathrm{Q}_{\mathrm{hp}+\mathrm{fcc}, \mathrm{c}} \mathrm{H}\end{array}$ & $\begin{array}{c}\text { Energy } \\
\text { use ratio } \\
\left(\mathrm{Q}_{\mathrm{e} . \mathrm{c}}+\mathrm{Q}_{\mathrm{e}, \mathrm{h}}+\mathrm{Q}_{\text {ah.h }}\right) \\
{ }_{(\%)} / \mathrm{Q}_{\text {load.h }} \\
(\%)\end{array}$ \\
\hline $\begin{array}{c}\text { 0600h-0559h } \\
\text { next day }\end{array}$ & $\varepsilon_{\mathrm{c}}$ & $\varepsilon_{\mathrm{h}}$ & $\varepsilon_{\mathrm{t} 1}$ & $\varepsilon_{\mathrm{t} 2}$ & $\eta$ & $r$ \\
\hline \multicolumn{7}{|l|}{ March } \\
\hline $10-11$ & 3.2 & 3.9 & 1.2 & 2.5 & 53.6 & 52.8 \\
\hline $11-12$ & 2.9 & 3.5 & 1.2 & 2.2 & 40.0 & 53.6 \\
\hline $12-13$ & 2.9 & 5.1 & 1.2 & 2.4 & 46.3 & 42.1 \\
\hline $13-14$ & 2.9 & 2.7 & 0.3 & 1.7 & 36.6 & 37.1 \\
\hline $14-15$ & 4.1 & 3.6 & 2.7 & 4.1 & 18.6 & 26.0 \\
\hline $15-16$ & 3.4 & 3.4 & 0.8 & 2.1 & 43.4 & 38.2 \\
\hline $16-17$ & 2.7 & 3.4 & 0.9 & 2.3 & 50.1 & 34.0 \\
\hline $17-18$ & 2.7 & 5.0 & 1.3 & 2.5 & 52.2 & 25.1 \\
\hline $18-19$ & 3.2 & 3.4 & 0.3 & 1.6 & 44.6 & 52.0 \\
\hline $19-20$ & 2.4 & N.M & N.M & N.M & 26.7 & N.M \\
\hline $20-21$ & 2.7 & 4.3 & 1.9 & 3.7 & 31.0 & 37.8 \\
\hline $21-22$ & 14.5 & 4.8 & 4.6 & 4.6 & 3.4 & 39.0 \\
\hline $22-23$ & 2.9 & 5.2 & 1.2 & 1.9 & 44.0 & 41.8 \\
\hline $23-24$ & 3.0 & 4.2 & 0.9 & 1.8 & 39.7 & 36.4 \\
\hline $24-25$ & - & 5.7 & 5.7 & 5.7 & 0.0 & 69.5 \\
\hline $25-26$ & 3.0 & 4.3 & 1.1 & 1.9 & 40.3 & 52.0 \\
\hline $26-27$ & 2.9 & 5.9 & 1.4 & 2.3 & 34.4 & 41.0 \\
\hline $27-28$ & 2.9 & 5.5 & 1.3 & 2.3 & 37.9 & 37.8 \\
\hline $28-29$ & 3.0 & 5.5 & 1.1 & 2.0 & 56.9 & 37.6 \\
\hline $29-30$ & 3.1 & 5.1 & 1.3 & 2.3 & N.M & 33.9 \\
\hline $30-31$ & 3.1 & 6.4 & 0.7 & 1.8 & N.M & 36.1 \\
\hline April & & & & & 0.0 & N.M \\
\hline $31-1$ & N.M & N.M & N.M & N.M & N.M & 36.0 \\
\hline $1-2$ & 3.3 & 5.6 & 1.2 & 2.3 & 35.4 & 52.9 \\
\hline $2-3$ & 2.6 & 5.5 & 0.5 & 1.6 & 30.3 & 51.9 \\
\hline $3-4$ & 2.1 & 6.0 & 0.4 & 2.0 & 35.2 & 62.5 \\
\hline $4-5$ & 2.1 & 4.4 & 1.2 & 2.3 & 22.1 & 58.2 \\
\hline $5-6$ & 2.7 & 4.6 & 1.1 & 1.9 & 29.3 & 63.9 \\
\hline $6-7$ & 2.7 & 4.3 & 0.8 & 1.7 & 33.7 & 66.3 \\
\hline $7-8$ & 2.5 & 4.3 & 1.0 & 1.9 & 30.4 & 58.1 \\
\hline $8-9$ & 2.9 & 4.6 & 0.8 & 1.7 & 31.3 & \\
\hline Average & 3.3 & 4.6 & 1.4 & 2.4 & 35.1 & 45.5 \\
\hline
\end{tabular}

N.M : Not Measured 


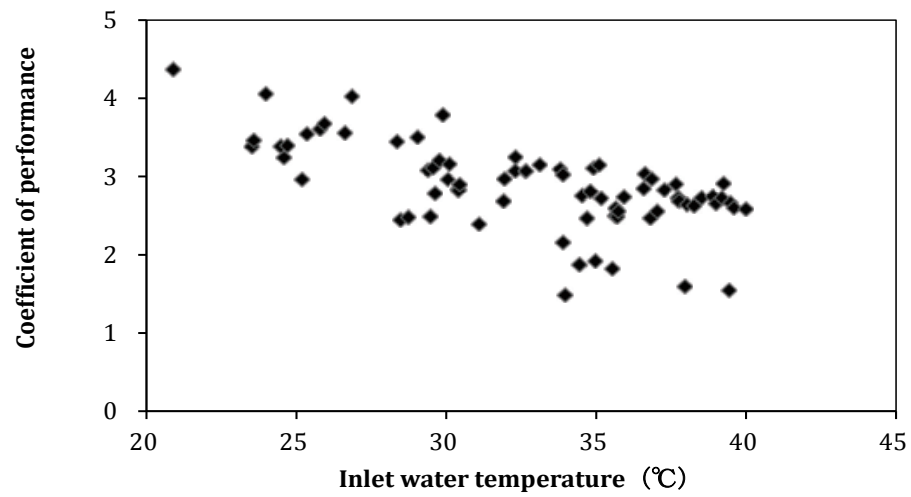

Fig. 3. Coefficient of performance of the heat pump as affected by the inlet water temperature during cooling operation. Each value represents the hourly average.

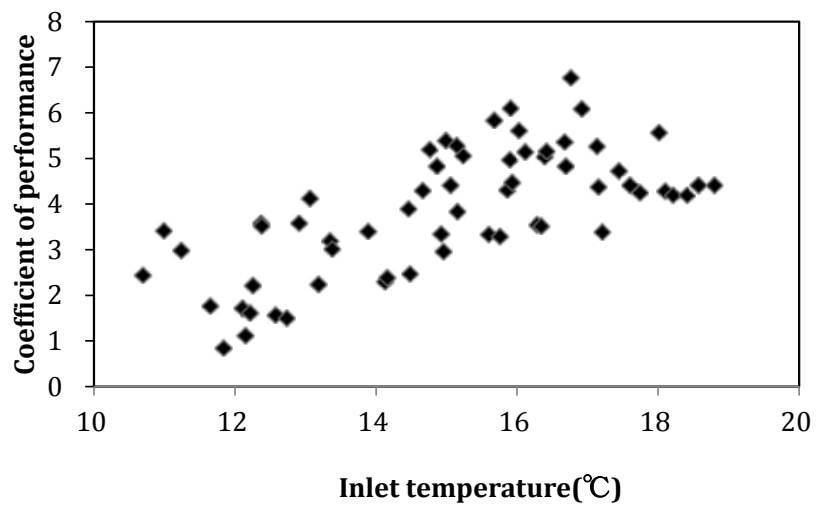

Fig. 4. Coefficient of performance of the heat pump as affected by the inlet water temperature during heating operation. Each value represents the hourly average.

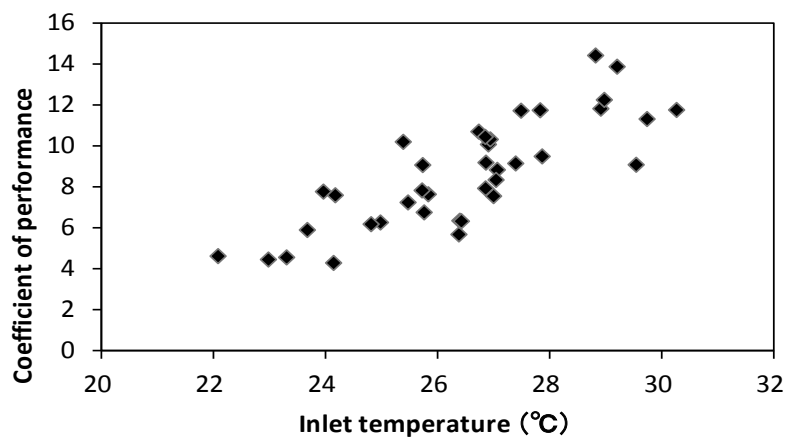

Fig. 5. Coefficient of performance of the fan coil unit as affected by inlet water temperature during heating operation. Each value represents the hourly average. 

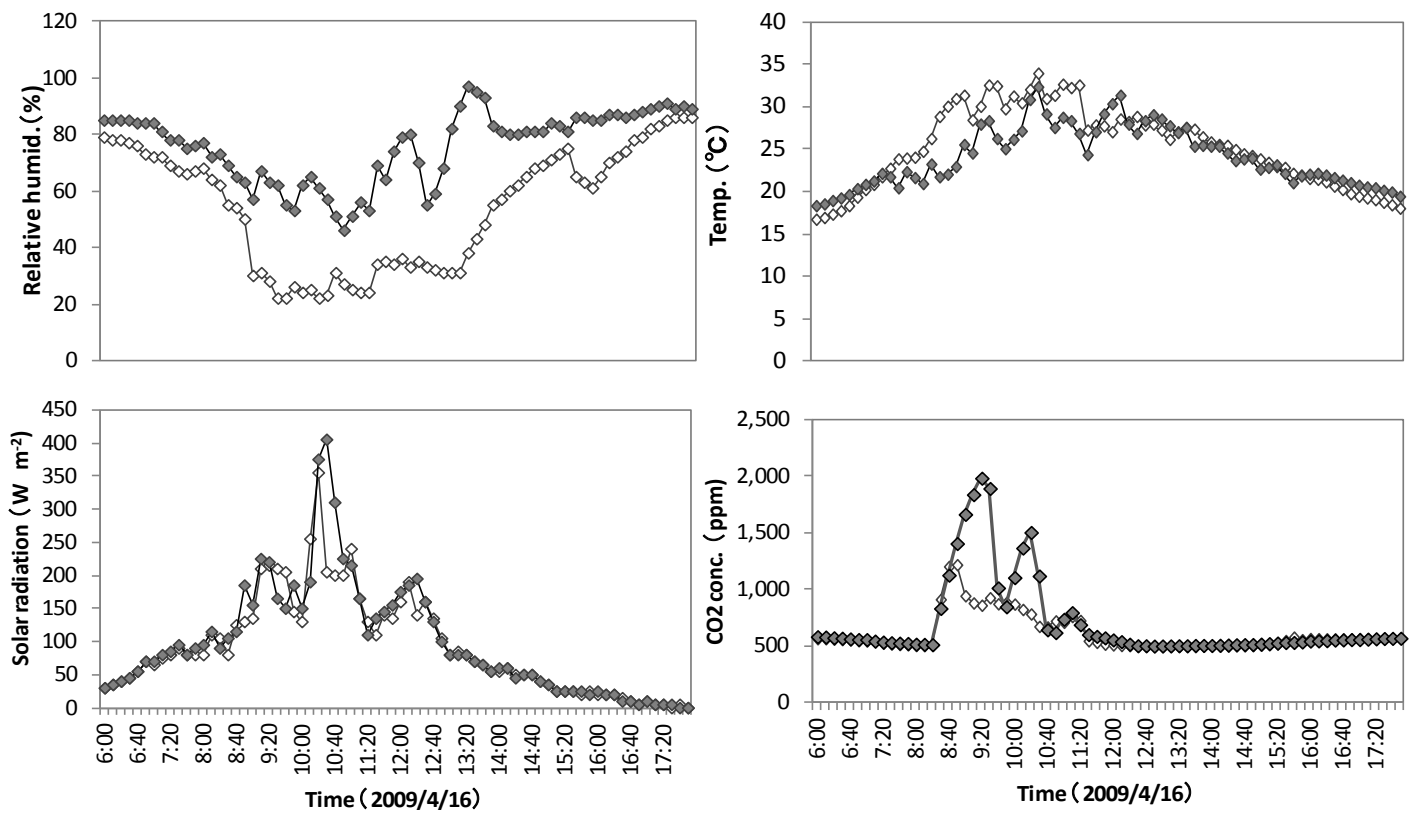

Fig. 6. Time course of (a) relative humidity, (b) solar radiation, (c) air temperature, and (d) $\mathrm{CO}_{2}$ concentration in $\mathrm{G}_{\mathrm{ct}}(\diamond)$ and $\mathrm{G}_{\mathrm{ex}}(\diamond)$ on 16 April 2009. A solid line on the graph (c) indicates the ventilation temperature $\left(30^{\circ} \mathrm{C}\right)$.

On the other hand, for the COP of the fan coil unit, as the inlet water temperature decreased, the COP decreased (Fig. 5).

\subsection{Energy use ratio}

The energy used in $\mathrm{G}_{\mathrm{ex}}$ was $45.5 \%$ of that used in $\mathrm{G}_{\mathrm{ct}}$, giving a $54.5 \%$ saving (Table 4 ).

\subsection{Changes in $\mathrm{CO}_{2}$ and humidity}

The cooling capacity of the experimental system was insufficient to completely control the air temperature rises, and ventilation was necessary on 13 days in March and 8 days in April. On April 14, a typical fine, warm day, as the solar radiation increased, the air temperature increased (Fig. 6b, c). The experimental system delayed the air temperature rise, so the ventilation started later and its duration was shorter in $\mathrm{G}_{\mathrm{ex}}$ than in $\mathrm{G}_{\mathrm{ct}}$. As a result, the $\mathrm{RH}$ and $\mathrm{CO}_{2}$ concentration were both higher in $G_{e x}$ than in $G_{c t}$ (Fig. 6a, d). This means the amount of $\mathrm{CO}_{2}$ released from the house was larger in $G_{\mathrm{ct}}$ than in $\mathrm{G}_{\mathrm{ex}}$. So the effectiveness of $\mathrm{CO}_{2}$ enrichment in $G_{e x}$ was higher than that in $G_{c t}$.

\section{Discussion}

Kozai et al. (1983) developed a system to collect and store heat from a greenhouse in a water tank by use of an air-water heat exchanger. The average COP of their system was 2.4. Hayashi et al. (1983) used a similar system that also released heat at night by use of a heat pump. The average COP during heating was 1.9. Oguni (1982) used a heat pump in the daytime to dehumidify the air, and used the heat held in the retained water for heating via the heat pump. The COP during heating was 2.3. In all cases, as the energy that is used to collect the heat increased, the COP during heating decreased.

In our experiment, to increase the cooling and heating capacity while minimizing the power consumption, we combined a low-power fan coil unit with a heat pump. During cooling (heat collection), the design favored the use of the heat pump, achieving capture of $34 \%$ of the heat, but at a high cost of electricity used, giving a COP $\left(\varepsilon_{t 1}\right)$ of only 1.5. Even when we added in the heat released from the soil surface to the air, the COP $\left(\varepsilon_{\mathrm{t} 2}\right)$ reached only 2.2. Considering the results of earlier reports and our experiment, we conclude that the collection of excess heat during the daytime for use at night is ineffective for saving energy.

The use of cooling to limit the ventilation time has been studied in the Netherlands (Heuvelink et al., 
2008; de Zwart, 2008; Qian et al., 2011). Minimizing the ventilation time extended the time during which $\mathrm{CO}_{2}$ concentration and high humidity could be maintained and thus increased yield (Yasuba et al., 2011). Ventilation introduces cooler, drier air, decreasing humidity and increasing water vapor pressure. Humidity in the greenhouse has a large effect on the photosynthetic rate, and higher humidity increases the photosynthetic rate by delaying stomatal closure (Yabuki and Miyagawa, 1970). Keeping the humidity high by fogging in the daytime increased the yields of tomato and strawberry by $>20 \%$ (Iwasaki et al., 2011) . The $\mathrm{CO}_{2}$ concentration and $\mathrm{RH}$ were higher in $\mathrm{G}_{\mathrm{ex}}$ than in $\mathrm{G}_{\mathrm{ct}}$. Although we did not investigate the growth or yield of the crop, we are certain that the photosynthetic rate will improve by using our environmental control system.

Many air-air heat pumps are used in commercial greenhouses, especially in rose production, so we must also compare the energy efficiency of our system with these. Air-air heat pumps had a reported average COP during heating of 3.5 in Morioka (Kawashima et al., 2008) and 3.0 in Miyagi (Iwasaki et al., 2010). We obtained an average COP during heating $\left(\varepsilon_{\mathrm{h}}\right)$ of 4.7 . We also obtained an average COP during cooling $\left(\varepsilon_{\mathrm{c}}\right)$ of 3.3, but there is little comparable information on airair heat pumps. Data from an air conditioner manufacturer's technical manual (Mitsubishi Electric, 2011) show that when the outside air temperature is $\leq 10^{\circ} \mathrm{C}$ and the inside temperature is $20^{\circ} \mathrm{C}$ (wet bulb), the COP is calculated to be 4.5 .

Our results show that the COP of the heat pump changes widely with water temperature in both cooling and heating operations. If we set the upper limit of the water temperature during cooling at $30-35^{\circ} \mathrm{C}$ and the lower limit at about $15^{\circ} \mathrm{C}$, the COP could increase greatly. We could improve it further by optimizing the volume of the heat storage tank, the preset values of water temperature, and the method of operating the heat pump, fan coil unit, and soil-water heat exchanger.

The use of a ground-source heat pump (Bakiri, 2010; Benli, 2011) or an air-water heat pump relying on groundwater (Iwasaki et al., 2010) has attracted attention. Takakura (2008) reported that in the Netherlands, they used an aquifer for seasonal heat storage and for cooling and heating the air in greenhouses. The temperature of groundwater is almost constant throughout the year, offering a more stable COP that is usually higher than that of air-air heat pumps. The COP of a heat pump using underground water in Miyagi was 3.9 (Iwasaki et al., 2010). To improve the operation of our environmental control system, we should be able to modify it to use groundwater when the water temperature in the heat storage tank moves outside the optimum range.

The effectiveness of any environmental control system should be evaluated on the consumption of energy, cost of production, and emission of $\mathrm{CO}_{2}$ per kilogram of produce, because the retained heat should increase the yield. In future work, we will grow crops and relate any improvements in yield to the costs of cooling.

\section{References}

Bakiri, K., 2010: Evaluation of the performance of a ground-source heat-pump system with series GHE (ground heat exchanger) in the cold climate region. Energy., 35, 3088-3096.

Benli, H., 2011: Energetic performance analysis of a ground-source heat pump system with latent heat storage for a greenhouse heating. Energy Conserv. Manage., 52, 581-589.

de Zwart, H. F., 2008: Overall energy analysis of (semi) closed greenhouses. Acta Hortic., 801, 811817.

Hayashi, M., Kozai, T., Nakamura, J., and Watanabe, I., 1983: Applications of a heat pump to greenhouse environmental control-(1) Heating system. J. Agric. Meteorol. 38, 379-387 (in Japanese with English abstract).

Heuvelink, E., Bakker, M., Marcelis, L. F. M., and Raaphorst, M., 2008: Climate and yield in a closed greenhouse. Acta Hortic., 801, 1083-1092.

Iwasaki, Y., Miura, S., and Ootsuki, Y., 2011: Effect of increase in humidity in the greenhouse on growth and yield of tomato and strawberry in forcing culture. J. Jpn. Soc. Hortic. Sci., 10 (Suppl. 2) , 455 (in Japanese).

Iwasaki, Y., Yoshida, C., and Aizawa, M., 2010: Comparison the energy efficiency between groundwater heat pump and air-air heat pump for greenhouse heating. Proc. 2010 Ann. Meet. Jpn. Soc. Agric. Biol. Env. Eng. Sci., Kyoto, 162-163 (in Japanese).

Kawashima, N., 1991: Present situation and problems of $\mathrm{CO}_{2}$ enrichment in a greenhouse. J. Agric. Meteorol., 47, 177-182 (in Japanese).

Kawashima, H., Takaichi, M., Baba, M., Yasui, K., and Nakano, Y., 2008: Effects of energy saving and 
the reduction of carbon dioxide emissions with a hybrid-heating system using an air-to-air heat pump for greenhouse heating. Bull. Natl. Inst. Veg. Tea Sci., 7, 27-36 (in Japanese with English abstract) .

Kawashiro, H., Tsuchiya, K., Sakiyama, H., and Udagawa, U., 2009: Effects of low-concentration carbon dioxide supplementation on fruit yield and economic value of cucumber on forced culture. Hortic. Res., $\mathbf{4}$, 445-449.

Kozai, T., Takeuchi, H., Okazaki, S., Watanabe, I., Hayashi, M., and Sida, K., 1983: Thermal properties of a solar greenhouse with in-house heat storage and heat exchange water tanks for nighttime heating. $J$. Agric. Meteorol., 38, 361-369 (in Japanese with English abstract) .

MAFF, 2009: Installation Situations of Greenhouse for Horticulture. Agricultural Production Bureau, Ministry of Agriculture, Forestry, and Fisheries, Tokyo, pp. 70-89.

Mitsubishi Electric, 2011: Technical manual of package air-conditioner for middle range, p. 107. http://wwwl7.mitsubishielectric.co.jp/wink_doc/com mon_files/TEC_MANUAL/M-P0575.pdf（accessed Jul. 3 2012) .

Oguni, K., 1982: A study on heat pumps for greenhouses.
J. Agric. Meteorol., 37, 317-322 (in Japanese) . Qian, T., Dieleman, J. A., Elings, A., De Gelder, A., Marcelis, and L. F. M., Kooten, O. V., 2011: Comparison of climate and production in closed, semiclosed and open greenhouses. Acta Hortic., 893, 807-814.

Shoji, K., Sasaki, T., Hino, Y., Suzuki, N., and Takahashi, N., 1982: Practical trial on effective utilization of solar heating for vegetable culture under structure in the cold region. Bull. Miyagi Pref. Hortic. Exp. Stn., 4, 17-41 (in Japanese).

Takakura, T., 2008: Long-term strategy for horticulture in the Netherlands. Agric. Hortic., 83, 151-1156.

Yabuki, K., and Miyagawa, H., 1970: Studies on the effect of wind speed upon the photosynthesis-(2) The relation between wind speed and photosynthesis. J. Agric. Meteorol., 26, 137-141 (in Japanese with English abstract).

Yasuba, K., Suzuki, K. Sasaki, H., Higashide, T., and Takaichi, M., 2011: Fruit yield and environmental condition under integrative environmental control for high yielding production at long-time culture of tomato. Bull. Natl. Inst. Veg. Tea Sci., 10, 85-93 (in Japanese with English abstract). 